\title{
DOMESTICACIÓN DE DOS ESPECIES DE LA FLORA PATAGÓNICA PARA USO ORNAMENTAL, Anemone multifida y Gaultheria mucronata*
}

\section{DOMESTICARON OF TWO SPECIES OF THE PATAGONIAN FLORA FOR ORNAMENTAL USE, Anemone multifida AND}

\section{Gaultheria mucronata}

\author{
Manzano, E. '; Mansilla, A. ${ }^{1}$; Seemann P. ${ }^{2}$; Schiappacasse, F. ${ }^{3}$; Musalem, M. ${ }^{4}$, Riedemann, P. ${ }^{5}$ \\ 'Centro de La Trapananda, Universidad Austral de Chile, Portales 73, Coyhaique. \\ E-mail: emanzano@uach.cl \\ ${ }^{2}$ Facultad de Ciencias Agrarias, Universidad Austral de Chile, Casilla 567, Valdivia. \\ ${ }^{3}$ Facultad de Ciencias Agrarias, Universidad de Talca, Casilla 747, Talca. \\ 'Vivero Pumahuida, Carretera San Martín 7021, Santiago.

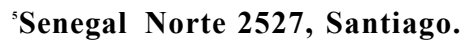

\section{INTRODUCCIÓN Y OBJETIVOS}

El desarrollo de nuevos cultivos ornamentales y florales ha alcanzado gran auge debido a las perspectivas de mercado que este rubro ofrece a nivel internacional. La búsqueda y manejo de nuevas especies nativas ha tomado gran interés. En Diciembre de 2001, el Centro Trapananda comenzó un proyecto cuyo objetivo central fue estudiar y manejar especies nativas que presentaban características para ser incorporadas en el uso paisajístico. Dos especies que formaron parte de este estudio manifestaron su potencial en este aspecto. Anemone multifida, perteneciente a la familia Ranunculaceae, conocida con el nombre de Anémona o Centella, presenta una abundante floración y número de varas lo cual la hace interesante para utilizar en macetas y jardines. Gaultheria mucronata, perteneciente a la familia Ericaceae, presenta vistosos frutos y follaje coriáceo, su potencial radica en su utilización como follaje, maceta y uso industrial de sus frutos.

Los objetivos de trabajo fueron recolectar material vegetal durante el crecimiento activo y receso de las plantas; estudiar la morfología, fenología y aspectos reproductivos de las especies; estudiar métodos de multiplicación como propagación el estaquillado, división y la propagación mediante semillas; para finalmente aplicar manejo agronómico a las especies (po- das, fertilizaciones, manejo de enfermedades).

\section{MATERIAL Y MÉTODO}

Se efectuaron recolecciones de material vegetal desde sus ambientes naturales de crecimiento, los cuales fueron identificados y caracterizados física y químicamente. Las colectas fueron el medio para obtener propágulos para iniciar los estudios de propagación. En Anemone multifida se aplicó el método de propagación mediante división de rizomas obteniéndose una tasa promedio de propagación de 5 a 7 nuevos individuos. En el caso de la propagación mediante semilla se obtuvo un $89 \%$ de germinación con semilla recolectada durante la temporada. A partir de este método se puede obtener plantas que florecen el primer año.

\section{RESULTADOS Y DISCUSIÓN}

Para G. mucronata se utilizó el método de propagación mediante estaquillado, donde se pueden obtener sobre $90 \%$ de enraizamiento de esquejes. La aplicación de AIB mejora la calidad de rizogénica de los esquejes (longitud y grado de enraizamiento). La propagación mediante semilla requiere de la aplicación de un tratamiento pre-germinativo con remojo de la semilla por 15 días en agua fría y frío por 8 semanas, de esta forma se logra alcanzar un 
$51 \%$ de germinación. A partir de las evaluaciones realizadas, se obtuvieron protocolos de multiplicación y fichas técnicas de manejo para cada especie.

Ambas especies también fueron descritas a partir de sus características morfológicas, altura de planta alcanzada, largo y número de tallos florales en el caso de Anémona, tipo de inflorescencia, entre otros. Estos resultados podrán ser usados para comparar las variaciones fenotípicas en futuros estudios de mejoramiento.

Hoy en día se cuenta con material vegetal ho- mogéneo en calidad y cantidad suficientes para iniciar una segunda fase de estudio. *

\section{BIBLIOGRAFÍA}

BANNISTER, P. 1990. Seed germination in Gaultheria antipoda, G. depressa and Pernettya macrostigma. New Zealand J. of Botany 28:357-358.

MASCO, M.; OLIVA, G.; KOFALT, R.; HUMANO, G. 1998. Flores de la Patagonia Austral. Convenio INTA-Consejo Agrario Provincial-Universidad Nacional de la Patagonia Austral. Río Gallegos, S.C., Argentina. 48p.

* Financiamiento mediante Proyecto FIA/Centro de La Trapananda-UACh C01-1-A-086.

Agro Sur 34 (1-2): 19-20 2006

PROPAGACIÓN VEGETATIVA DE Baccharis magellanica, UNA ESPECIE NATIVA DE LA REGIÓN DE MAGALLANES Y ANTÁRTICA CHILENA*

\title{
VEGETATIVE PROPAGATION OF Baccharis magellanica, A NATIVE SPECIES FROM MAGALLANES AND THE CHILEAN ANTARCTIC REGION
}

\author{
Yagello J.', Vera M.', Massardo F. ${ }^{2}$, Dollenz O. ${ }^{3}$ y Musalem M. \\ 'Instituto de la Patagonia-Universidad de Magallanes. Av. Bulnes 01890, Casilla 113D, Punta \\ Arenas. E-mail: julio.yagello@umag.cl. \\ ${ }^{2}$ Parque Etnobotánico Omora-Sede Puerto Williams- Universidad de Magallanes. \\ ${ }^{3}$ Departamento de Ciencias y Recursos Naturales- Universidad de Magallanes. \\ ${ }^{4}$ Vivero Pumahuida, Santiago.
}

\section{INTRODUCCIÓN}

El romerillo enano (Baccharis magellanica) es una Asteraceae nativa que crece en la XII Región hasta la Isla Navarino $\left(55^{\circ} \mathrm{S}\right)$, límite de distribución en el rango austral. Esta especie se considera ornamental debido a su reducido tamaño, ramificación abierta y hábito de crecimiento rastrero que lo convierten en una especie deseable como cubrepiso adecuado para incluir en macizos, borduras, orillas de camino y control de taludes. Además, tiene aroma agradable durante la época de floración por lo que se puede considerar su ubicación en el jardín en zonas de tránsito o de descanso, entradas, terrazas y bordes de ventanas.
El objetivo de este trabajo es determinar las técnicas de propagación vegetativa y domesticación de B. magellanica.

\section{MATERIAL Y MÉTODO}

La colecta de material de B. magellanica se realizó en las cercanías del Parque Nacional Torres del Paine $\left(51^{\circ} 23^{\prime} \mathrm{S}\right)$ en junio 2004, octubre 2004 y enero 2005, en sitios expuestos alterados por sobrepastoreo o quema de bosques. Se escogieron 15 individuos plus y se seleccionaron ramas con crecimiento rastrero con evidente potencial de formación de raíces adventicias. Las ramillas se mantuvieron con humedad hasta su esquejado final. Los esquejes obtenidos se mantuvieron 